\title{
Generating Function for the Spherical Functions on a Gelfand Pair of Exceptional Type
}

\author{
Shigeru WATANABE \\ Sophia University \\ (Communicated by T. Nagano)
}

Let $X$ be a non-compact Riemannian symmetric space of rank 1 . Then it is known that $X=G / K$, where $G$ is a connected simple Lie group with finite center, $K$ is a maximal compact subgroup of $G$, and if $G=K A N$ is an Iwasawa decomposition, we have $\operatorname{dim} A=1$. From the classification theory, it is known that $X$ is either one of the classical hyperbolic spaces corresponding to the groups $S O_{0}(1, n), S U(1, n)$ and $S p(1, n)$ over the fields $R, C$ and $\boldsymbol{H}$ or the exceptional space corresponding to the exceptional simple group $F_{4(-20)}$, the so-called Cayley hyperbolic plane. Let $M$ be the centralizer of $A$ in $K$, then the Martin boundary $K / M$ of $X=G / K$ is not a symmetric space, except for the case of real numbers. But, as is well known, $(K, M)$ is a Gelfand pair, i.e. the convolution algebra of functions on $K$ bi-invariant by $M$ is commutative. A theory of the corresponding spherical functions is given in an expose of Takahashi [5] for the classical case, while the exceptional case is treated in [6].

In the case of real hyperbolic spaces, the space $K / M$ is the usual unit sphere $S^{n-1}$ in $\boldsymbol{R}^{n}$, and we have the classical theory of spherical harmonics; the zonal spherical functions are given essentially by the Gegenbauer polynomials $C_{p}^{(n-2) / 2}$ and we have the classical generating function expansion:

$$
\left(1-2 t x+t^{2}\right)^{-(n-2) / 2}=\sum_{p=0}^{\infty} C_{p}^{(n-2) / 2}(x) t^{p}, \quad-1 \leq x \leq 1, \quad-1<t<1,
$$

which can be considered also as giving a generating function for the zonal spherical functions of the space $S O(n) / S O(n-1)$. In the papers [7], [8], we have shown that similar constructions are possible also in the other classical cases. The purpose of the present paper is to give a generating function in the exceptional case.

In what follows, we will follow the notations of [6].

The compact group $K$ acts transitively on $\left\{F_{2}^{u}+F_{3}^{v} ; u, v \in O,|u|^{2}+|v|^{2}=1\right\} \cong S^{15}$ and the isotropy group of $F_{2}^{1}$ is the subgroup $M \subset L$, that is $K / M \cong S^{15}$, and it's identification is given by $k M \mapsto k F_{2}^{1}=F_{2}^{u}+F_{3}^{v}$. See $\S 4$ iv) in [6]. 
A zonal spherical function $\varphi$ of $K / M$ depends only on $\operatorname{Re}(u)$ and $|u|$, and there uniquely exists a pair of nonnegative integers $(p, q)$ such that

$$
\varphi\left(k F_{2}^{1}\right)=c_{p q} C_{p}^{3}\left(\frac{\operatorname{Re}(u)}{|u|}\right)|u|_{2}^{p} F_{1}\left(-q, p+q+7 ; p+4 ;|u|^{2}\right),
$$

where

$$
c_{p q}=\frac{(-1)^{q}(p+4)_{q}}{(4)_{q}}\left[C_{p}^{3}(1)\right]^{-1} .
$$

See the formula (12), (13) of $\S 6$ in [6]. From now on, we denote $\varphi$ by $\varphi_{p q}$. When we denote $\left\{f * \varphi_{p q} \mid f \in L^{2}(K / M)\right\}$ by $H_{p q}, H_{p q}$ is $K$-irreducible and moreover $H_{k}^{(16)} \cong$ $\bigoplus_{p+2 q=k} H_{p q}, L^{2}(K / M)=\bigoplus_{p, q=0}^{\infty} H_{p q}$, where $H_{k}^{(16)}$ is the space of restrictions to $S^{15}$ of harmonic polynomials on $\boldsymbol{R}^{16}$ which are homogeneous of degree $k$. The main theorem is

THEOREM 1. If $k \in K, w \in O,|w|=1$ and $0 \leq r<1$, then

$$
\int_{S O(7)}\left[1-2 r \operatorname{Re}(\alpha(u) w)+r^{2}\right]^{-7} d \alpha=\sum_{p, q=0}^{\infty} \gamma_{p q} \varphi_{p q}\left(k F_{2}^{1}\right) C_{p}^{3}(\operatorname{Re}(w)) r^{p+2 q},
$$

where $d \alpha$ is the normalized Haar measure on $S O(7)$ and

$$
\begin{gathered}
K F_{2}^{1}=F_{2}^{u}+F_{3}^{v}, \\
\gamma_{p q}=\frac{2 p+6}{\Gamma(p+q+4)} \frac{(7)_{p+q}(4)_{q}}{q !} .
\end{gathered}
$$

The series on the right hand side converges absolutely and uniformly for $k, w$ and $r \leq \rho$ for each $\rho<1$.

Proof. The function $f$ defined by

$$
f(\xi)=\left\|\xi-e_{1}\right\|^{-14}, \quad \xi=\left(\xi_{1}, \xi_{2}\right) \in O^{2}, \quad e_{1}=(1,0) \in O^{2},
$$

is harmonic on $|\xi|<1$. Therefore we have the following expansion which converges uniformly on every compact subset of $|\xi|<1$,

$$
f(\xi)=\sum_{v=0}^{\infty} h_{v}(\xi)
$$

where the function $h_{v}$ is a harmonic polynomial on $R^{16}$ which is homogeneous of degree $v$.

We define the $R$-linear map $a$ as follows:

$$
a: F_{2}^{u^{\prime}}+F_{3}^{v^{\prime}} \mapsto\left(u^{\prime}, v^{\prime}\right) \in O^{2} .
$$

For $k \in K$ and $0 \leq r<1$, if we put $\xi=r a\left(k F_{2}^{1}\right)$, then we obtain that

$$
f\left(r a\left(k F_{2}^{1}\right)\right)=\sum_{\nu=0}^{\infty} r^{v} h_{v}\left(a\left(k F_{2}^{1}\right)\right) .
$$


The function $k \mapsto f\left(r a\left(k F_{2}^{1}\right)\right)$ ) is $M$ bi-invariant because $e_{1}=a\left(m F_{2}^{1}\right)$ for all $m \in M$ and $\left\|a\left(l k F_{2}^{1}\right)\right\|=\left\|a\left(k F_{2}^{1}\right)\right\|$ for all $l \in L$ (see Lemma 2 of $\S 3$ in [6]). So the functions $k \mapsto h_{v}\left(a\left(k F_{2}^{1}\right)\right)$ are also $M$ bi-invariant. Thus there exist constants $a_{p q} \in \boldsymbol{R}$ such that

$$
h_{v}\left(a\left(k F_{2}^{1}\right)\right)=\sum_{p+2 q=v} a_{p q} \varphi_{p q}\left(k F_{2}^{1}\right)
$$

So we see that

$$
f\left(r a\left(k F_{2}^{1}\right)\right)=\sum_{v=0}^{\infty} r^{v} \sum_{p+2 q=v} a_{p q} \varphi_{p q}\left(k F_{2}^{1}\right)
$$

From the function equations for $\varphi_{p q}$, for $k^{\prime} \in L$

$$
\int_{M} f\left(r a\left(k^{\prime} m k F_{2}^{1}\right)\right) d m=\sum_{\nu=0}^{\infty} r^{\nu} \sum_{p+2 q=v} a_{p q} \varphi_{p q}\left(k^{\prime} F_{2}^{1}\right) \varphi_{p q}\left(k F_{2}^{1}\right),
$$

where $d m$ is the normalized Haar measure on $M$. We now define $k^{\prime} \in L$ by

$$
\left(\alpha_{1}, \alpha_{2}, \alpha_{3}\right)=k^{\prime} \in D_{4} \text {, where } \alpha_{1}(u)=w u, \alpha_{2}(u)=u w \text { and } \alpha_{3}(u)=\bar{w} u \bar{w}
$$

(see Lemma 2 of $\S 3$ in [6]). If we write $m=(\tilde{\alpha}, \alpha, \kappa \tilde{\alpha})(\alpha \in S O(7), \tilde{\alpha} \in S O(8))$, then

That is to say

$$
k^{\prime} m k F_{2}^{1}=F_{2}^{\alpha(u) w}+F_{3}^{\bar{w}(\alpha \tilde{\alpha}(v)) \bar{w}} \text {. }
$$

$$
a\left(k^{\prime} m k F_{2}^{1}\right)=(\alpha(u) w, \bar{w}(\kappa \tilde{\alpha}(v)) \bar{w})
$$

So we see that

$$
f\left(r a\left(k^{\prime} m k F_{2}^{1}\right)\right)=\left[1-2 r \operatorname{Re}(\alpha(u) w)+r^{2}\right]^{-7} .
$$

From (2), we can conclude that

$$
\begin{aligned}
\int_{S O(7)} & {\left[1-2 r \operatorname{Re}(\alpha(u) w)+r^{2}\right]^{-7} d \alpha } \\
= & \sum_{v=0}^{\infty} r^{v} \sum_{p+2 q=v} a_{p q}\left[C_{p}^{3}(1)\right]^{-1} C_{p}^{3}(\operatorname{Re}(w)) \varphi_{p q}\left(k F_{2}^{1}\right)
\end{aligned}
$$

We now determine the constants $a_{p q}$ using the following formula. See [3].

If $\mu>\lambda>0$, then we have

$$
C_{v}^{\mu}(t)=\sum_{q=0}^{[v / 2]} \gamma_{q}^{(v)}(\mu, \lambda) C_{v-2 q}^{\lambda}(t)
$$

where

$$
\gamma_{q}^{(v)}(\mu, \lambda)=\frac{(\lambda+v-2 q)}{(\lambda+v-q)} \frac{(\mu)_{v-q}}{(\lambda)_{v-q}} \frac{(\mu-\lambda)_{q}}{q !}
$$


Putting $k=e$, i.e. $u=1$, in (3), we obtain that

$$
\left(1-2 r \operatorname{Re}(w)+r^{2}\right)^{-7}=\sum_{v=0}^{\infty} r^{v} \sum_{p+2 q=v} a_{p q}\left[C_{p}^{3}(1)\right]^{-i} C_{p}^{3}(\operatorname{Re}(w))
$$

So we see that

$$
\sum_{p+2 q=v} a_{p q}\left[C_{p}^{3}(1)\right]^{-1} C_{p}^{3}(\operatorname{Re}(w))=C_{v}^{7}(\operatorname{Re}(w))
$$

and moreover

$$
a_{p q}\left[C_{p}^{3}(1)\right]^{-1}=\frac{2 p+6}{\Gamma(p+q+4)} \frac{(7)_{p+q}(4)_{q}}{q !} .
$$

Finally, it follows from the definitions of $\varphi_{p q}$ that $\left|\varphi_{p q}\left(k F_{2}^{1}\right)\right| \leq \varphi_{p q}\left(F_{2}^{1}\right)=1$ for $k \in K$, which implies the last assertion.

The formula (1) means that the zonal spherical functions $\varphi_{p q}$ appear as the coefficients in the expansion of the left hand side of (1) by the powers of $r$ and by the spherical functions of $S^{7} \cong\{u \in O ;|u|=1\}$. So we can consider that (1) gives a generating function for the functions $\varphi_{p q}$. This interpretation for generating function is similar to the classical cases. See [7], [8].

\section{References}

[1] A. Erdélyi, W. Magnus, F. Oberhettinger and F. G. Tricomi, Higher Transcendental Functions, Vol. 2, McGraw-Hill (1953).

[2] J. Faraut and K. Harzallah, Deux Cours d'Analyse Harmonique, Birkhäuser (1987).

[3] L. K. HUA, Harmonic Analysis of Functions of Several Complex Variables in the Classical Domains, Amer. Math. Soc. (1963).

[ 4 ] K. D. Johnson and N. R. WALLACH, Composition series and intertwining operators for the spherical principal series I, Trans. Amer. Math. Soc. 229 (1977), 137-173.

[5] R. TAKAHASH, Lectures on harmonic analysis in hyperbolic spaces (in preparation).

[6] R. TAKAHASH, Quelques résultats sur l'analyse harmonique dans l'espace symétrique non compact de rang 1 du type exceptionnel, Analyse Harmonique sur les Groupes de Lie. II, Lecture Notes in Math. 739 (1979), 511-567, Springer.

[7] S. Watanabe, Generating functions for the spherical functions on some classical Gelfand pairs, Proc. Japan Acad. Ser. A 68 (1992), 140-142.

[8] S. WATANABE, Generating functions and integral representations for the spherical functions on some classical Gelfand pairs, J. Math. Kyoto Univ. 33 (1993), 1125-1142.

Present Address:

Center for Mathematical Sciences, The University of Aizu,

Tsuruga, Ikki-Machi, Aizu-Wakamatsu City, Fukushma, 965-80 Japan. 